\title{
Kepler mission highlights
}

\author{
William J. Borucki ${ }^{1}$, David G. Koch ${ }^{1}$ and the Kepler Team \\ ${ }^{1}$ Space Science Directorate, Mail Stop 244-30, NASA Ames Research Center Moffett Field, CA \\ 94035 \\ email: William.J.Borucki@NASA.gov
}

\begin{abstract}
During the first 33.5 days of science-mode operation of the Kepler Mission, the stellar flux of 156, 000 stars were observed continuously. The data show the presence of more than 1800 eclipsing binary stars, over 700 stars with planetary candidates, and variable stars of amazing variety. Analyses of the commissioning data also show transits, occultations and light emitted from the known exoplanet HAT-P7b. The depth of the occultation is similar in amplitude to that expected from a transiting Earth-size planet and demonstrates that the Mission has the precision necessary to detect such planets. On 15 June 2010, the Kepler Mission released most of the data from the first quarter of observations. At the time of this data release, 706 stars from this first data set have exoplanet candidates with sizes from as small as that of the Earth to larger than that of Jupiter. More than half the candidates on the released list have radii less than half that of Jupiter. Five candidates are present in and near the habitable zone; two near super-Earth size, one similar in size to Neptune, and two bracketing the size of Jupiter. The released data also include five possible multi-planet systems. One of these has two Neptune-size (2.3 and 2.5 Earth-radius) candidates with near-resonant periods as well as a super-Earth-size planet in a very short period orbit.
\end{abstract}

Keywords. space vehicles, telescopes, planetary systems, techniques: photometric

\section{Introduction}

Kepler is a Discovery-class mission designed to determine the frequency of Earth-size planets in and near the habitable zone (HZ) of solar-type stars. In Sections 2 and 3, the Mission is summarized and the data described. Discoveries are described in Sections 4 through 9 . Sections 10 and 11 discuss the availability of the data and provide a summary.

\section{Kepler Mission description}

The instrument is a wide field-of-view photometer designed to monitor over 150,000 stars for at least 3.5 years with enough precision to find Earth-size planets in the habitable zone. The instrument design is shown in Figure 1. The instrument has a 0.95 meter aperture and a spectral bandpass with a throughput greater than $40 \%$ from 430 to 820 $\mathrm{nm}$ and a $5 \%$ response cutoff at 423 and $897 \mathrm{~nm}$. The shortwave cutoff reduces stellar variability due to Calcium $\mathrm{H}$ and $\mathrm{K}$ lines. The instrument monitors a single large area of the sky centered on R.A. $=19^{h} 22^{m} 40^{s}$ and decl. $=44^{\circ} 30^{\text {min }} 00^{\text {sec }}$ and is in a 53-week long heliocentric orbit to allow continuous observations of the targets to avoid missing transits

The focal plane of the Schmidt-type telescope contains 42 CCDs with a total of 95 megapixels that cover 115 square degrees of sky. Kepler was launched into an Earthtrailing heliocentric orbit on 6 March 2009, finished its commissioning on 12 May 2009, and is now in science operations mode. Further details of the Kepler Mission and instrument can be found in Koch et al. (2010a), Jenkins et al. (2010a), and Caldwell et al. (2010). 


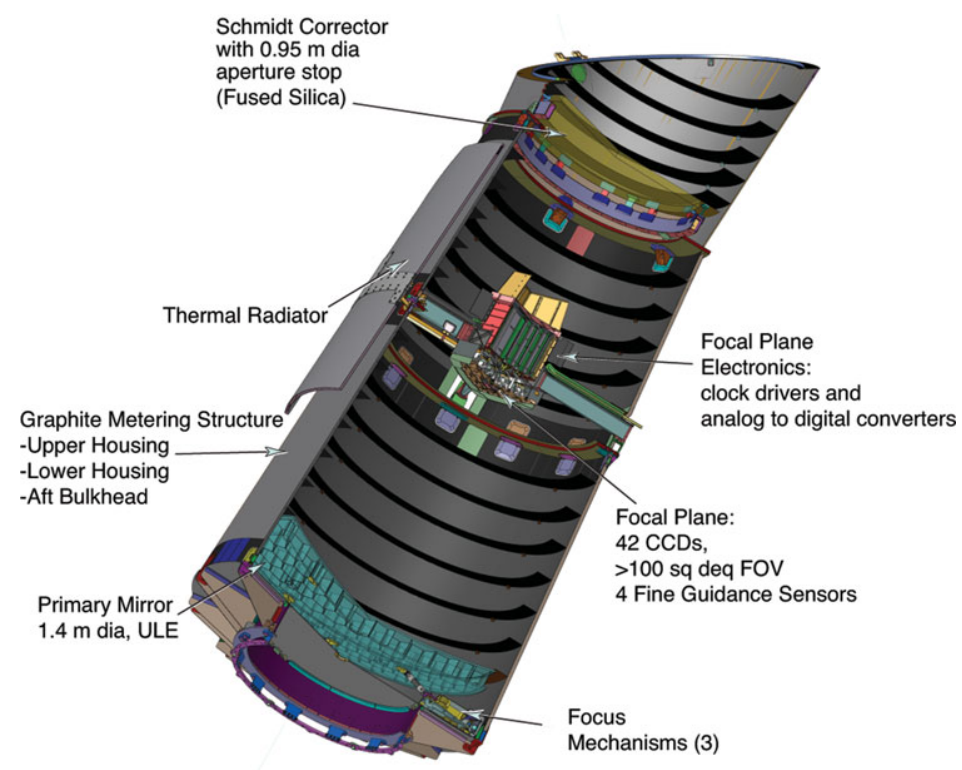

Figure 1. Diagram of the Kepler Instrument

\section{Data description}

During the first 33.5 days of science-mode operation, 156, 097 stars were observed. Five new exoplanets with sizes between 0.3 and 1.6 Jupiter radii and orbital periods from 3.2 to 4.9 days were confirmed by radial velocity observations (Borucki et al. 2010; Koch et al. 2010a; Dunham et al. 2010; Jenkins et al. 2010b; Latham et al. 2010). Later, three additional planets were confirmed using a combination of RV observations and variations in the times of transits (Holman et al. 2010; Torres et al. 2011). Most of the results shown in this paper are based on the first data segment taken at the beginning of science operations on 13 May 2009 UT and finished on 15 June 2009 UT; a 33.5day segment (labeled Q1). Additional data were used to confirm the discovery of three planets detected by transit timing variations and 9.7 days of data (labeled $Q 0$ ) taken during commissioning of spacecraft and instrument were used to analyze the exoplanets known to be in the Kepler field-of-view (FOV).

The target stars are primarily main sequence dwarfs chosen from the Kepler Input Catalog (KIC). These were chosen to maximize the number of stars that were both bright and small enough to show detectable transit signals for small planets in and near the HZ (Batalha et al. 2010). Most stars were in the magnitude range $9<K p<16$. Data for all stars are recorded at a cadence of one per 29.4 minutes (hereafter, long cadence, or LC). Data for a subset of 512 stars are also recorded at a cadence of one per 58.5 seconds (hereafter, short cadence or SC), sufficient to conduct asteroseismic observations needed for measurements of the stars' size, mass, and age. The results presented here are based only on LC data. For a full discussion of the LC data and their reduction, see Jenkins et al. (2010b,c). See Gilliland et al. (2010) for a discussion of the SC data.

\section{Detection of Radiation from HAT-P-7b}

The Q0 data were analyzed to investigate the transit characteristics of the previously known giant transiting exoplanet HAT-P-7b (Pal et al. 2008). A comparison of the 

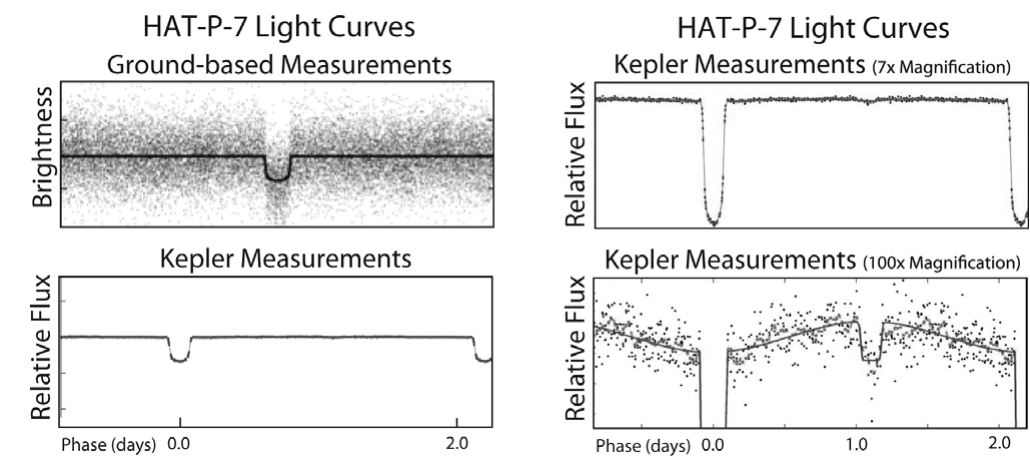

Figure 2. Kepler observations of exoplanet HAT-P-7b. Left panel upper: Ground based observations. Left panel lower: Scatter of the data points in the Kepler data is within the line thickness. Kepler precision is 100 times better than that from ground-based observations. Right panel; Scale expanded 7 and 100 times. The transit is off scale on the bottom half of the right panel, but the occultation and the variation of light from the combination of starlight and planet emission are clearly visible.

ground based observations (upper left hand panel) with those from Kepler is presented in the lower left hand panel of Figure 1. The great improvement in photometric precision obtained for space-based operation compared to the ground-based measurement is evident in the left hand panel. In the right hand panel, the Kepler data for HAT-P-7b show a smooth rise and fall of light from the planet as it orbits its star, punctuated by a drop of $130 \pm 11 \mathrm{ppm}$ in flux when the planet passes behind its star. We interpret this as the phase variation of the dayside thermal emission plus reflected light from the planet as it orbits its star and is occulted. The depth of the occultation is similar in significance to the detection of a transiting Earth-size planet for which the mission was designed.

Kepler's photometric detection of the optical phase curve and occultation of HAT-P-7b confirms the prediction of Raymond et al. (2006). The depth of the occultation and the shape and amplitude of the phase curve indicate that HAT-P-7b could have a strongly absorbing atmosphere and inhibited advection to the night side. If the planet has a completely absorbing atmosphere, its dayside temperature is estimated to be $2650 \pm 100 \mathrm{~K}$. The position in phase of the occultation is consistent with zero orbital eccentricity, as expected from the radial velocity variation.

\section{Validation of Discoveries}

After the beginning of science operations, several thousand candidates were recognized, but most of these were quickly shown to be false positive events, usually energetic-particle events or data breaks. A comprehensive program was initiated to confirm the candidates as planets before announcing them to the public.

The search for planets starts with a search of the time series of each star for a pattern that exceeds a detection threshold commensurate with a non-random event. Observed patterns of transits consistent with those from a planet transiting its host star are labeled "planetary candidates." Those that were at one time considered to be planetary candidates but subsequently failed some consistency test are labeled "false positives". After passing the consistency tests listed below, and only after a review of all the evidence by the entire Kepler Science Team, does the candidate become a confirmed or validated exoplanet.

List of Tests Conducted Prior to Radial Velocity Observations; 


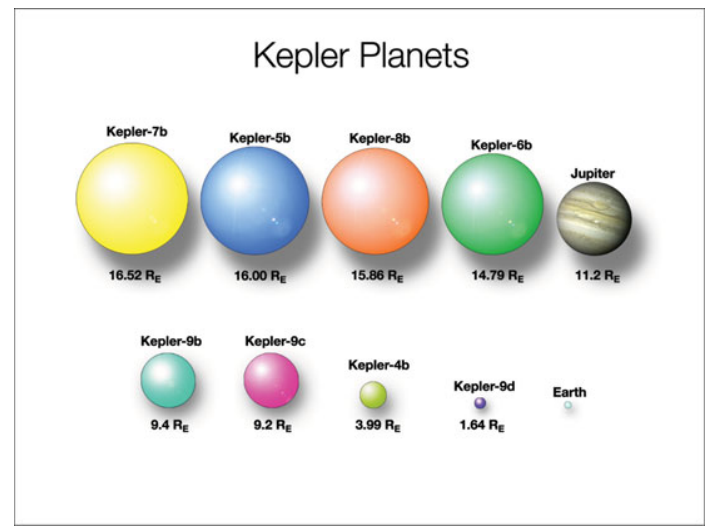

Figure 3. Planets discovered and confirmed by the Kepler Mission in 2010.

- $\mathrm{SNR}>7.1 \sigma$ to rule out statistical fluctuations

- At least three transits to confirm orbital periodicity

- Light curve depth, shape, and duration must be consistent with a Keplerian orbit

- Search for secondary eclipses

- Centroid analysis to identify signals from background stars

\section{Confirmation of Early Discoveries}

After the first month of data were transmitted to ground and analyzed, only a few weeks remained to make radial velocity (RV) measurements before the star field set for ground-based observations. There was sufficient time during the Fall of 2009 to make the necessary RV observations to confirm five short-period planets; Kepler 4 through 8. See top row of Figure 3.

Kepler $-5 b,-6 b,-7 b$, and $-8 b$ are larger and less dense than Jupiter. Kepler $-8 b$ with a density of $0.17 \mathrm{gr} / \mathrm{cc}$ is one of the lowest density planets ever discovered. Kepler $-4 b$ is similar in mass and density $(1.91 \mathrm{gr} / \mathrm{cc})$ to Neptune $(1.67 \mathrm{gr} / \mathrm{cc})$, but with a stellar flux nearly a million times larger because it is only $0.046 \mathrm{AU}$ from it star. Its radiative equilibrium temperature is $1650 \mathrm{~K}$. Because of such high irradiation levels, such planets were expected to have a much lower density (Fortney 2008; Chabrier et al. 2009).

Although RV observations have been critical to the confirmation of most of these planets and to the determination of their masses and densities, the present-day RV precision of $1 \mathrm{~m} / \mathrm{s}$ for bright stars with sharp spectral lines is insufficient for low-mass planets in long period orbits around dim stars; i.e., for most of the planetary candidates found by the Kepler Mission.

The relative sizes of the measurement error bars in Figure 4 shows the increasing difficulty of confirming photometric detections as the planet mass decreases. For longer period orbits, less massive planets, and dimmer stars, the precision of the RV observations will usually be too low to confirm the photometrically discovered planets and therefore other methods must be employed for confirmation and validation.

For the case of a low-mass planet, the reflex orbital motion of the star will often be below the limit of detectability. However, under favorable circumstances, the distortion of stellar spectral lines caused by a transiting planets successively blocking Doppler shifted portions of the stellar surface during a transit (the Rossiter-McLaughlin effect) may produce an anomalous Doppler signal that is detectable even for the transit of an Earthsize planet (Gaudi et al. 2007; Welsh \& Orosz 2007). See Figure 5. Although the signal 


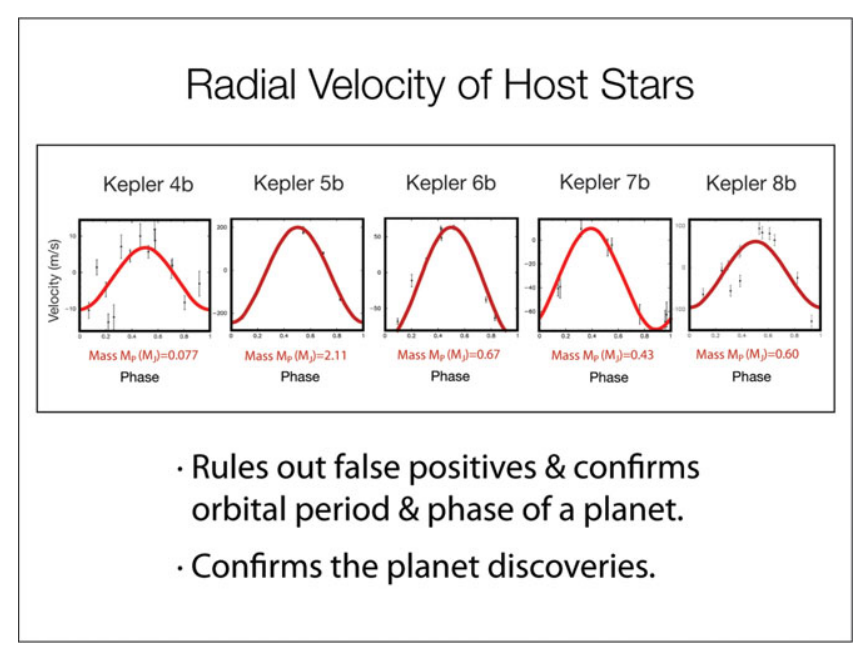

Figure 4. RV confirmation of first five Kepler planets.

cannot be used to derive the mass of the planet, it does rule out the possibility that a background binary is the cause of the signal. The Rossiter-McLaughlin effect (Winn et al. 2005) helped to confirm Kepler-8b (Jenkins et al. 2010b).

Another method used to confirm planets is the mutual gravitational interaction that causes variations in the transit times. The interactions are proportional to the planet masses and are strongest for resonant or near-resonant orbits. This situation allowed a three-planet system to be announced; Kepler-9b,-c, and $-d$ (Holman et al. 2010; Torres et al. 2011). The size distribution of the three planets is shown in the bottom panel of Figure 3. Only the two Saturn size planets are in near-resonant orbits and thus show strong transit timing variations. See Figure 6. The third planet Kepler-9d is in a 1.59 day period and not significantly perturbed by the two outer planets (Torres et al. 2011).

A comparison of the distributions shown in Figure 7 indicates that the majority of the candidates discovered by Kepler are Neptune-size (i.e., $3.8 R_{\oplus}$ ) and smaller; in contrast, the planets discovered by the transit method and listed in the Extrasolar Planets Encyclopedia (EPE) are typically Jupiter-size (i.e., 11.2R $R_{\oplus}$ ) and larger. This difference is understandable because of the difficulty in detecting small planets when observing through the Earth's atmosphere and because of the day/night cycle which causes most transits to be missed.

The Kepler results shown in Figure 7 imply that small candidate planets with periods less than 33.5 days are much more common than large candidate planets with periods less than 33.5 days and that the ground-based discoveries are potentially sampling only the upper tail of the size distribution (Gaudi et al. 2005; Gould et al. 2003). Note that for a substantial range of planet sizes, an $R^{-2}$ curve fits the Kepler data well. Because it is much easier to detect larger candidates than smaller ones, the result is robust; it implies that the frequency of planets decreases with the area of the planet, assuming that the false positive rate and other biases are independent of planet size for planets larger than 2 Earth radii.

\section{Characteristics of the Detected planets}

Most of the planets shown in Figure 8 have short orbital periods and are therefore quite hot; hotter than molten lava and self-luminous. As the mission duration increases, 


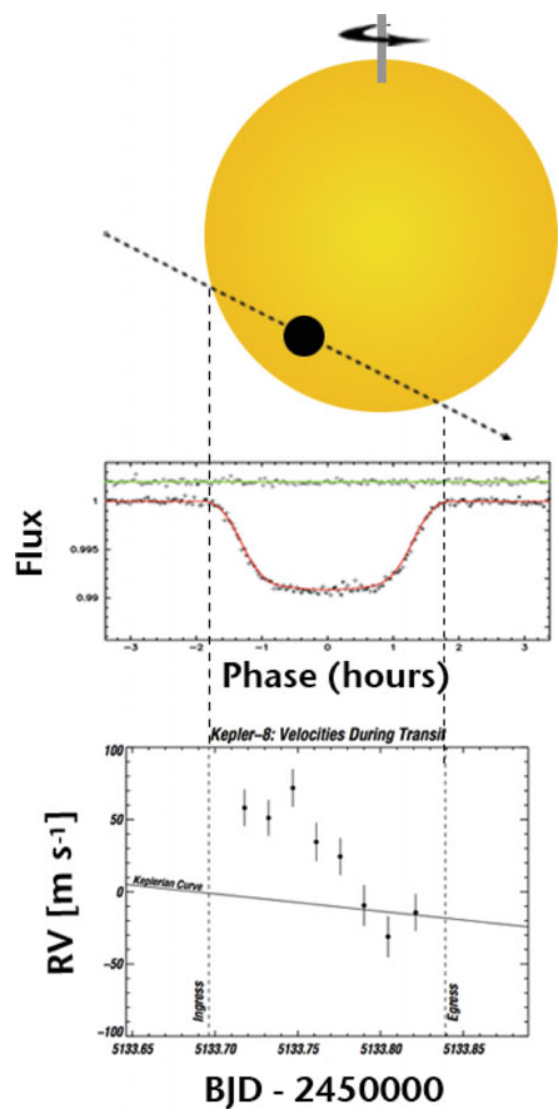

Figure 5. Rossiter-McLaughlin effect for Kepler-8b. Although the orbit plane is not perpendicular to the stellar spin axis, the transit shape is normal, but a strong asymmetrical shift in the spectral lines occurs.

planets with longer orbital period and cooler temperatures can be found and confirmed. The two Saturn-size planets (Kepler-9b and -9c) have orbital periods 19.2 days and 38.9 days and are substantially cooler. Figure 9 shows that four of the first five Kepler planets have densities less than that of a hydrogen-helium gas mixture of similar mass. Many of the planets discovered by ground-based observations (open circles) are consistent with the result. It is not known whether the calculation of the state properties of such mixtures must be revised or if it is the measurements that need revision.

Kepler $-9 b$ and $-9 c$ are the outer two planets and have temperatures lower than that of Mercury and Venus. The inner most planet Kepler-9d has a temperature well above the melting point of iron. It was validated by ruling out other explanations because the RV observations did not have sufficient precision to confirm this planet (Torres et al. 2011). It's density is undetermined and thus is not shown in figure 9 .

\section{Additional Highlights}

The four star clusters shown in Figure 8 are in the Kepler FOV and span a very large range of ages, temperatures, and rotation periods. An investigation is underway to use the Kepler measurements to better define the surface relating these parameters. When the study is complete and the surface calibrated, it should be possible to determine 


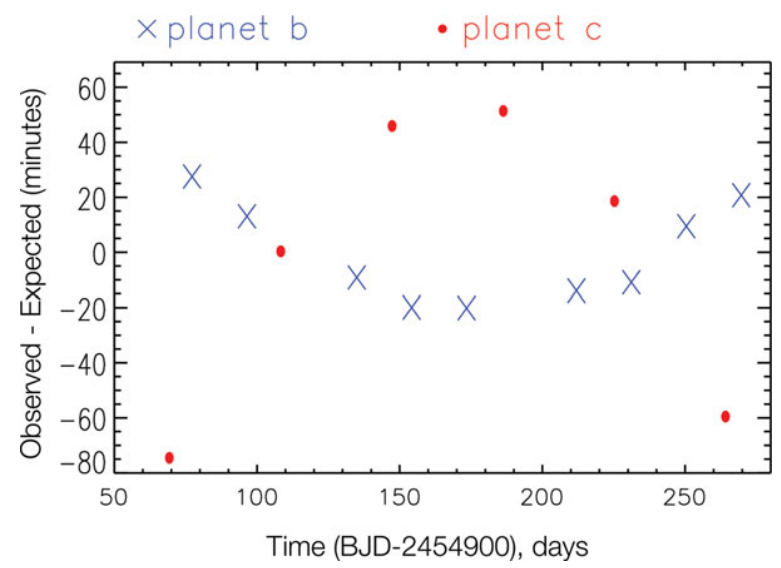

Figure 6. Mutual interactions among Kepler-9b and $-9 \mathrm{c}$ cause the observed transit times to vary.
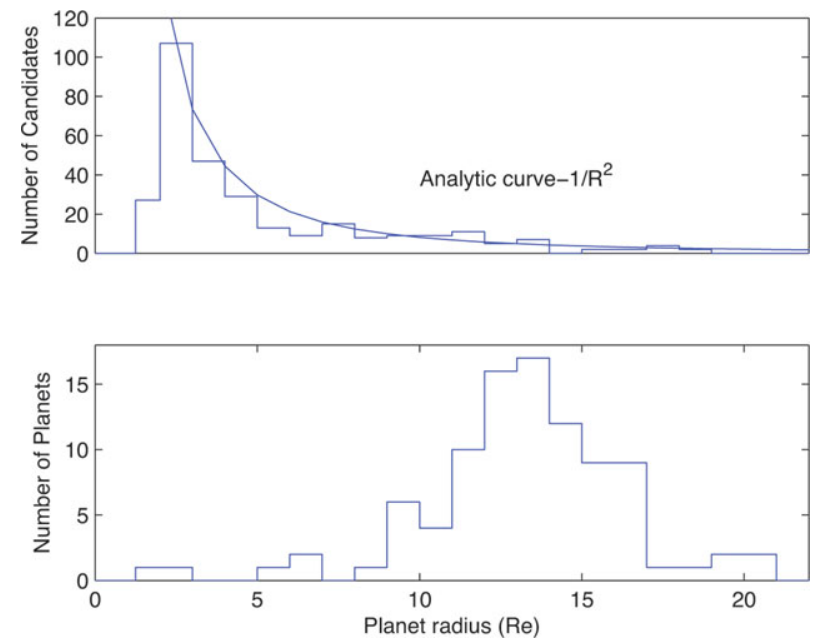

Figure 7. Size distribution. Upper panel: Kepler candidates. Lower Panel: Non-Kepler candidates planets discovered by the transit method and listed in the EPE as of 2010 December 7 (without Kepler planets).

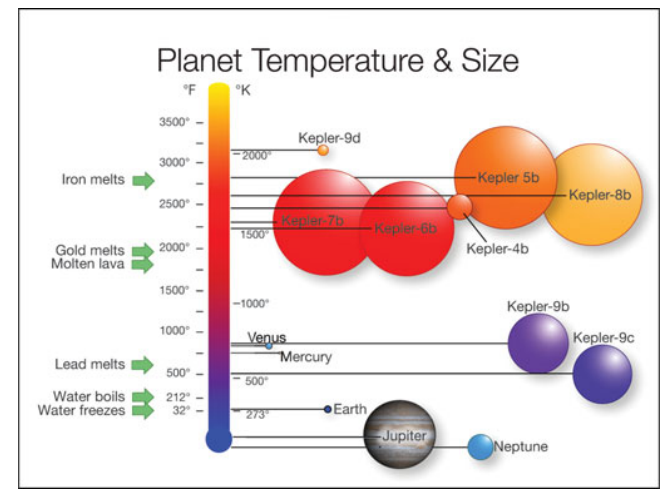

Figure 8. Relative sizes and temperatures of Kepler planets. Comparisons of candidate temperatures to melting points of common materials. 


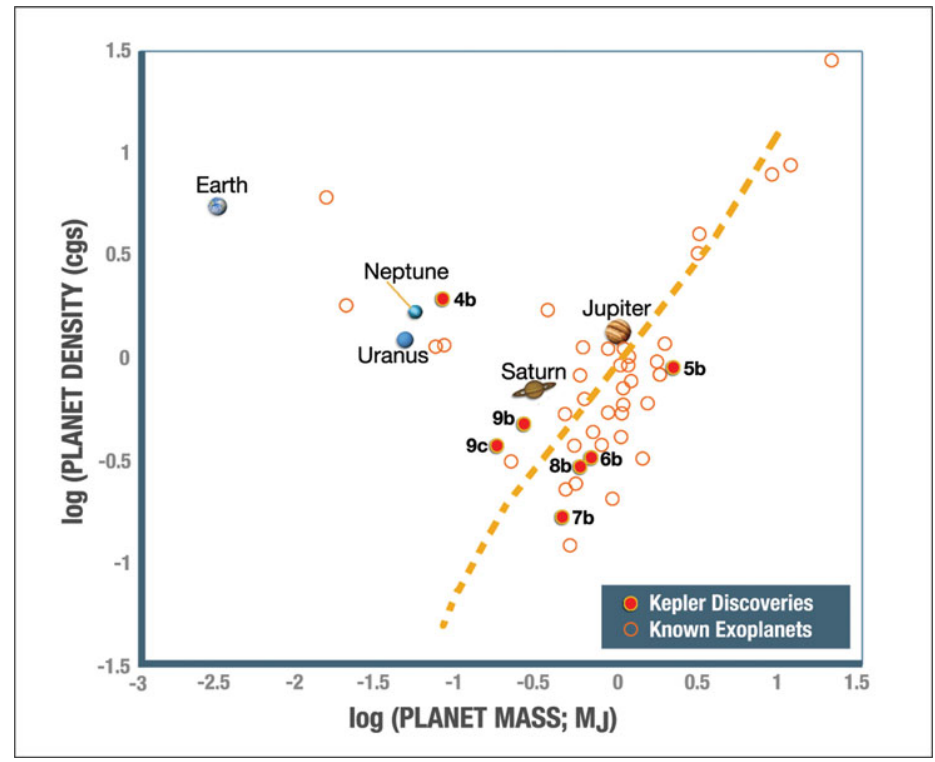

Figure 9. Comparison of confirmed planets with Solar system planets and expected density curve for gas giant planets. The dashed curve is a calculation of the density versus mass for a self gravitating hydrogen/helium sphere (Fortney 2008)

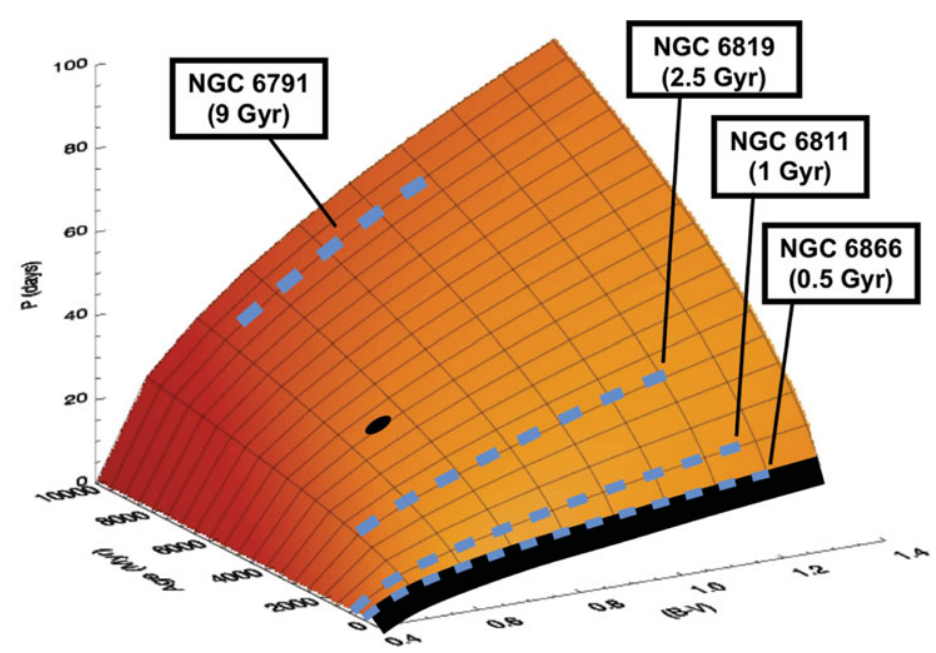

Figure 10. Expected relationship between stellar temperature, age, and rotation period. Black area marks the currently studied star clusters. Blue lines labels the four clusters being investigated using Kepler data.

stellar ages of field stars from measurements of their temperature and rotation period. The current relationship is tentative and based only on young clusters (marked in black) and the Sun's characteristics (Skumanich 1972; Barnes 2003).

The light curve shown in Figure 11 is one example of the many surprises that the Mission has produced. The observations indicate that the star temperature is $9400 \mathrm{~K}$ and the companion temperature is $12,200 \mathrm{~K}$. The size of the companion is 0.8 the radius of Jupiter. The light curves might be explained by an orbiting white dwarf, but white dwarfs are expected to be much smaller; approximately Earth-size. This example is one of two found in the first data set (Rowe et al. 2010). 

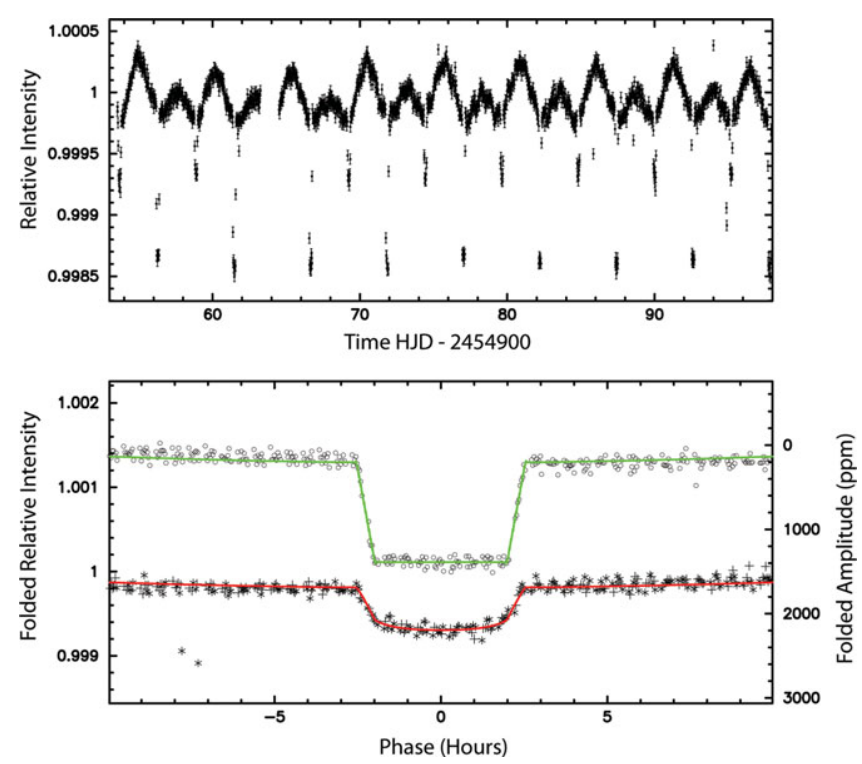

Figure 11. Light curve (upper panel) and folded light curves (bottom panel) showing 500 ppm transit and a $1300 \mathrm{ppm}$ occultation.

\section{Data Release}

At the one-year anniversary of the receipt of the first set of data from the beginning of science operations, the data for 156,097 stars covering the $Q 0$ and $Q 1$ periods became available to the public in June 2010, with two exceptions: 400 stars held back to allow completion of one season of observations by the Kepler team, and 2778 stars held back for the Guest Observers and Kepler Asteroseismic Science Consortium (KASC). These data will be released on 1 February 2011, and in November 2010 when the proprietary periods are complete, respectively. A total of 152,919 stars are currently available at several levels of processing at the Multi-Mission Archive at the Space Telescope Science Institute (MAST $\dagger$ ) for analysis by the community.

Summary of observations

- Over 700 stars with planetary candidates have been found.

- Characteristics and identification of 305 planetary candidates were released to the public in June 2010. All $Q 0$ through $Q 2$ data will be released for all candidates in February 2011.

- Hundreds of the remaining candidates were observed during the summer of 2010.

- 800 high precision RV measurements of 45 stars

- Active optics observations of 83 stars

- Speckle observations of 72 stars

- 750 reconnaissance spectra obtained for 550 stars

- Centroid-movement plots for 129 stars.

A list of false positive events found in the released data is also available at the MAST. The identification of the false positives should help the community avoid wasting observation resources.

Characterization of the candidates is based on the stellar magnitude, effective temperature, and surface gravity of the star taken from the KIC. Also listed in the MAST archive are the orbital period, epoch, and an estimate of the size of each candidate.

$$
\dagger \text { http : //archive.stsci.edu/kepler/kepler }{ }_{f} \text { ov/search. }
$$


When only one transit is seen in the data, the epoch and period are calculated using data obtained subsequently. More information on the characteristics of each star can be obtained from the KIC. Several of the target stars show more than one series of planetary transit-like events and therefore have more than one planetary candidate. These candidate multi-planet systems are of particular interest to investigations of planetary dynamics.

\section{Summary}

The Kepler instrument is functioning well and many planetary candidates are being discovered. Data for over 300 candidates have been released so that the community can independently confirm the planets. Data for an additional several hundred candidates will be released in February 2011 when the analysis of this summer's follow up are available and when more of the false positive events have been identified and removed.

\section{Acknowledgments}

Funding for this Discovery mission is provided by the NASA Science Mission Directorate.

\section{References}

Barnes S. A. 2003, ApJ, 586, 464

Batalha, N. M, et al. 2010, ApJL, 713, L109

Borucki, W. J., et al. 2009, Science, 325, 709

Borucki, W. J., et al. 2010, ApJL, 713, L126

Caldwell, D. A., et al. 2010, ApJL, 713, L92

Chabrier, G., et al. 2009, AIP Conf. Proc., 1094, 102

Dunham, E. W., et al. 2010, ApJ, 713, L136

Fortney, J. J. 2008 ASP Conf. Ser., 398, 405

Gaudi, B. S., et al. 2005, ApJ, 628, L73

Gaudi, B. S., et al. 2007, ApJ, 655, 550

Gould, A., et al. 2003, ApJL, 591, L53

Gilliland, R. L., et al. 2010, ApJL, 713, L160

Holman, M. J., et al. 2010, Science, 330, 51

Jenkins, J. M., et al. 2010, ApJL, 713, L87

Jenkins, J. M., et al. 2010, ApJ, 724, 1108

Jenkins, J. M., et al. 2010, ApJL, 713, L120

Koch, D. G., et al. 2010, ApJL, 713, L79

Koch, D. G., et al. 2010, ApJL, 713, L131

Latham, D. W., et al. 2010, ApJL, 713, L140

Pal, A., et al. 2008, ApJ, 680,1450

Raymond, S. N., et al. 2006, Science, 313, 1413

Rowe, J. F., et al. 2010, ApJL, 713, L150

Skumanich, A. 1972, ApJ, 171, 565

Torres, G., et al. 2010, ApJ, 727, 24

Welsh, W. F. \& Orosz, J. A. 2007, ASP Conf Ser., 366, 176

Winn, J. N., et al. 2005, ApJ, 631, 1215 